\title{
Reproductive organs regulate leaf nitrogen metabolism mediated by cytokinin signal
}

\author{
Daisuke Igarashi · Yoshihiro Izumi · \\ Yuko Dokiya $\cdot$ Kazuhiko Totsuka \\ Eiichiro Fukusaki · Chieko Ohsumi
}

Received: 12 August 2008/Accepted: 5 November 2008/Published online: 2 December 2008

(C) The Author(s) 2008. This article is published with open access at Springerlink.com

\begin{abstract}
The metabolism of vegetative organs in plants changes during the development of the reproductive organs. The regulation of this metabolism is important in the control of crop productivity. However, the complexity of the regulatory systems makes it difficult to elucidate their mechanisms. To examine these mechanisms, we constructed model experiments using Arabidopsis to analyze metabolic and gene expression changes during leaf-stage progression and after removal of the reproductive organs. Leaf gene expression levels and content of major amino acids, both of which decreased during leaf-stage progression, increased after removal of the reproductive organs. In particular, the levels of expression of cytokinin biosynthesis genes and cytokinin-responsive genes and the cytokinin content increased after removal of the reproductive organs. Analysis of plants with knockout of a cytokinin-biosynthetic gene (AtIPT3) and a cytokinin receptor gene (AHK3) indicated that glutamate dehydrogenase genes (GDH3) were regulated by cytokinin signaling. These data suggest that cytokinins regulate communication between reproductive and vegetative organs, and that $G D H 3$ is one target of the cytokinin-mediated regulation of nitrogen metabolism.
\end{abstract}

Electronic supplementary material The online version of this article (doi:10.1007/s00425-008-0858-2) contains supplementary material, which is available to authorized users.

D. Igarashi $(\bowtie) \cdot$ Y. Dokiya $\cdot$ K. Totsuka $\cdot$ C. Ohsumi Institute of Life Sciences, Ajinomoto Co., Inc.,

1-1, Suzuki-cho, Kawasaki-ku, Kawasaki 210-8681, Japan

e-mail: daisuke_igarashi@ajinomoto.com

Y. Izumi · E. Fukusaki

Department of Biotechnology, Graduate School of Engineering,

Osaka University, 2-1 Yamadaoka, Suita 565-0871, Japan
Keywords Amino acid biosynthesis - Arabidopsis . Cytokinin - Glutamate dehydrogenase .

Nitrogen metabolism $\cdot$ Sink-source regulation

$\begin{array}{ll}\text { Abbreviations } \\ \text { AAP } & \text { Amino acid permease } \\ \text { EOL } & \text { Excision of organs above the rosette leaves } \\ \text { GDH } & \text { Glutamate dehydrogenase } \\ \text { GOGAT } & \text { Glutamate 2-oxoglutarate aminotransferase } \\ \text { GS } & \text { Glutamine synthetase } \\ \text { iP } & \text { Isopentenyladenine } \\ \text { iPR } & \text { Isopentenyladenosine } \\ \text { IPT } & \text { Isopentenyl transferases } \\ \text { tZR } & \text { Trans-zeatin riboside }\end{array}$

\section{Introduction}

Plants utilize nitrate and ammonium as external nitrogen sources. Ammonium is the final form of inorganic nitrogen before the synthesis of organic nitrogen compounds, and it is assimilated into the glutamine amide group, which is then transferred to 2-oxoglutarate, yielding two molecules of glutamate by the concerted reaction of glutamine synthetase (GS) and glutamate synthase (GOGAT). The two major isoenzymes of GS are located in different subcellular compartments and display non-overlapping roles. In the photosynthetic tissues of many angiosperms, GS2, the plastid-located isoform, is responsible for the assimilation of ammonium derived from nitrate reduction and photorespiration (Ireland and Lea 1999). GS1, the cytosolic isoform, is the predominant enzyme in roots and nonphotosynthetic tissues and is much less abundant in green tissues (Ireland and Lea 1999). 
GOGAT is present in two distinct forms, one that uses reduced ferredoxin $(\mathrm{Fd}$; EC 1.4.7.1) as the electron donor and one that uses NADH (EC 1.4.1.14). The Fd-dependent enzyme is normally present and highly active in the chloroplasts of photosynthetic tissues, where it is able to utilize light energy as a supply of reductant. The NADH-dependent enzyme, which is present in various plastids, is located predominantly in non-photosynthesizing cells, where reductant is supplied by the pentose phosphate pathway (Bowsher et al. 2007).

The major function of GS2 and Fd-GOGAT in chloroplasts is in photorespiratory nitrogen metabolism (Leegood et al. 1995; Lea and Miflin 2003); the other GS/GOGAT species are important for normal growth and development (Yamaya et al. 2002; Yamaya and Oaks 2004).

A third enzyme, glutamate dehydrogenase (GDH; EC 1.4.1.2), catalyzes a reversible amination-deamination reaction, which can lead to either synthesis or catabolism of glutamate. GDH is responsible for the deamination of glutamate under normal conditions (Melo-Oliveira et al. 1996; Masclaux-Daubresse et al. 2006; Skopelitis et al. 2006, 2007; Miyashita and Good 2008).

Following the formation of glutamate, the $\alpha$-amino group can be transferred to a wide variety of 2-oxo acid acceptors to form amino acids, or can be transferred back to form glutamate when 2-oxoglutarate and other amino acids are available. The reactions are performed by the action of reversible aminotransferase (EC 2.6.1.x).

In senescing leaves, a large amount of ammonium is generated as a result of protein and nucleic acid hydrolysis, and it might be directly incorporated into glutamate by GDH and subsequently into glutamine by GS1 (Miflin and Habash 2002).

Leaf senescence is essential for the development of reproductive tissues, because it is characterized by a transition from nutrient assimilation to nutrient remobilization. Numerous studies have been carried out to define the mechanisms of regulation of leaf senescence, and it is suggested that some hormone signals, such as cytokinin, ethylene, salicylic acid, and jasmonate, and various environmental signals are involved in senescence (BuchananWollaston et al. 2003). Cytokinins have a particular profound effect on the longevity of plant organs (Gan and Amasino 1995; McCabe et al. 2001). For example, transgenic plants with increased cytokinin biosynthesis show delayed senescence and increased productivity (Gan and Amasino 1995). Numerous reports have indicated that cytokinins control nitrogen metabolism (Takei et al. 2004; Sakakibara et al. 2006), suggesting that the metabolic control of nitrogen and amino acid metabolism by cytokinins is important for leaf senescence and the remobilization of nutrients from source to sink organs.
Cytokinin biosynthesis and signal transduction mechanisms have recently been well characterized (Kakimoto 2003; Sakakibara 2006; To and Kieber 2007; Hirose et al. 2008). This information is useful for the analysis of the relationship of cytokinins to leaf senescence. For example, an Arabidopsis mutant with gain-of-function of the cytokinin receptor showed delayed senescence (Kim et al. 2006), indicating that cytokinin signaling is a key factor in the control of leaf senescence.

The key step in the biosynthesis of isopentenyladenine (iP)- and trans-zeatin (tZ)-type cytokinins is isopentenylation by ATP and ADP isopentenyl transferases (IPTs) (Kakimoto 2001; Haberer and Kieber 2002; Miyawaki et al. 2004; Sakakibara 2006). Analyses of IPT genes have revealed that AtIPT3, which is predominantly expressed in the phloem, is upregulated by macronutrients such as nitrate, sulfate, and phosphate (Miyawaki et al. 2004; Takei et al. 2004; Hirose et al. 2008). Cytokinin application significantly induces or represses the genes encoding macronutrient transporters (Brenner et al. 2005). The differential regulation of transporter genes by cytokinins and upregulation of IPT genes by macronutrients imply that cytokinins play a key role as a feedback signal to maintain a balance in the acquisition of macronutrients (Hirose et al. 2008). These previous reports indicate that cytokinins play an important role in nutrient metabolism in the leaves, but the relationship between cytokinin signaling and leaf nitrogen metabolism is unclear.

In monocarpic plants, the reproductive structures often govern the senescence of the whole plant, with especially striking effects on the leaves. In soybean, for example, by the time pod filling is well under way, leaf growth is coming to a close. Removal of the pods before the seeds mature prevents the completion of senescence and death of the plant (Nooden 1980). In Arabidopsis, the longevity of the whole plant is under correlative control of the reproductive structures (Nooden and Penney 2001). These reports suggest that metabolic regulation during the progression of vegetative growth is controlled by systems that regulate the source-sink balance.

Our aim in the present study was to examine the mechanisms of regulation of leaf nitrogen metabolism during leaf development. In addition, by analysis of the amino acid contents and comprehensive gene expression data, we investigated the sink-source regulation of leaf metabolism.

\section{Materials and methods}

Plant materials and growth conditions

Arabidopsis thaliana L. (Col-0, purchased from Lehle seeds, Round Rock, TX, USA) cultures were grown at a 
photosynthetically active radiation of $100 \mu \mathrm{mol} \mathrm{m}{ }^{-2} \mathrm{~s}^{-1}$, a photoperiod of 14:10-h L:D, a temperature of $23^{\circ} \mathrm{C}$ and $60 \%$ humidity, unless otherwise indicated. Plants were grown on rockwool moistened with nutrient solution containing $2.5 \mathrm{mM}$ potassium phosphate $(\mathrm{pH} 5.7), 2.5 \mathrm{mM}$ $\mathrm{NH}_{4} \mathrm{NO}_{3}, 2 \mathrm{mM} \mathrm{Ca}\left(\mathrm{NO}_{3}\right)_{2}, 2 \mathrm{mM} \mathrm{MgSO}_{4}, 50 \mu \mathrm{M} \mathrm{Na}-\mathrm{Fe}-$ EDTA, $10 \mu \mathrm{M} \mathrm{NaCl}, 70 \mu \mathrm{M} \mathrm{H}_{3} \mathrm{BO}_{3}, 14 \mu \mathrm{M} \mathrm{MnCl}_{2}, 1 \mu \mathrm{M}$ $\mathrm{ZnSO}_{4}, 0.5 \mu \mathrm{M} \mathrm{CuSO}_{4}, 10 \mathrm{nM} \mathrm{CoCl} 2$, and $0.2 \mu \mathrm{M} \mathrm{Na}-$ $\mathrm{MoO}_{4}$. The nutrient solution was renewed once a week.

Information on plants with knockout of AtIPT3, AHK2, and AHK3 (atipt3-2, ahk2-2, and ahk3-3, respectively) has been given in previous reports (Higuchi et al. 2004; Miyawaki et al. 2006).

EOL refers to excision of the main stem and axillary shoots above the rosette leaves. The main stem contains the cauline leaves and lateral buds. The axillary shoots contain buds located at the axils of the rosette leaves and small rosette leaves emerging from the axils of the axillary buds (Tatematsu et al. 2005). Under our growth conditions, plants bolted about 30 days after the seeds had been sown onto rockwool. EOL was performed 7 days after bolting. For the analysis of main vein gene expression, the vein was cut out of the leaf body to a width of about $5 \mathrm{~mm}$, containing the petiole and the cells around the main vein.

\section{Gene expression analysis}

For reverse transcription-polymerase chain reaction (RTPCR), total RNA was isolated from the leaves and main veins, using an RNeasy plant mini kit (Qiagen). First-strand cDNA synthesis was conducted with an oligo(dT)12-18 primer (GE Healthcare) and reverse-transcriptase SuperScript II (Invitrogen). Real-time PCR reactions were performed with an Applied Biosystems Prism 7500 sequence detector. The primers used for real-time PCR were as follows (in parentheses): RBCS-1B (5'-GCTTCACTG ATGCTTAAATCCTTT-3' and 5-'ATTTACATTGACCAT ACAAAATTGG-3'), AtIPT3 (5'-GGCGTGATCAGTTG TAACCA- $3^{\prime}$ and $5^{\prime}$-CCGTCACCATCACCAATAAG- ${ }^{\prime}$ ), GDH3 (5'-TGCTGATTCAATCGATCCTG- $3^{\prime}$ and $5^{\prime}$-TTG ATGACTCCCCCTAGAGC-3'), ARR5 (5'-AAACGCGCA AAGATCTGAGT- $3^{\prime}$ and $5^{\prime}$-AAGCCGAAAGAATCAG GACA- $3^{\prime}$ ), ARR6 (5'-TGTTCACTCGCAGCTCAAAC- $3^{\prime}$ and $5^{\prime}$-GATCCAGTCACGGAATTTGC-3'), ARR7 (5'-AA ACCGGTGAAGCTAGCAGA- $3^{\prime}$ and $5^{\prime}$-GCATTCCTCA GCTTCATTCC- $\left.3^{\prime}\right)$, and ACT2 (5'-GGTAACATTGTGCT CAGTGGTGG- $3^{\prime}$ and $5^{\prime}$-GGTGCAACGACCTTAATCTT CAT- $\left.3^{\prime}\right)$.

Real-time RT-PCR reactions were performed in duplicate with $0.9 \mu \mathrm{M}$ of each primer and $1 \times$ SYBR green PCR master mix (Applied Biosystems) in a $50 \mu \mathrm{L}$ volume. Relative differences were determined by the $\Delta \Delta \mathrm{Ct}$ method described by the manufacturer.
Measurement of amino acid content

Amino acids were extracted from leaves in $80 \%$ ethanol at $80^{\circ} \mathrm{C}$. After evaporation, dried samples were dissolved in $0.02 \mathrm{M} \mathrm{HCl}$. Amino acid content was determined with an L-8800 amino acid analyzer (Hitachi). Briefly, amino acids separated by cation-exchange chromatography were detected spectrophotometrically after post-column reaction with ninhydrin reagent (Noguchi et al. 2006).

Labeling of RNA probes and hybridization to an Arabidopsis GeneChip

Labeling and hybridization of RNA were performed in accordance with the manufacturer's instructions. Briefly, ATH1 Arabidopsis GeneChips (Affymetrix) were used to measure changes in gene expression levels. Total RNA was converted into cDNA, which was in turn used to synthesize biotinylated cRNA. The cRNA was fragmented into smaller pieces and then hybridized to the GeneChips. After hybridization, the chips were automatically washed and stained with streptavidin phycoerythrin using an Affymetrix Fluidics Station.

Microarray data were extracted from the scanned GeneChip images and analyzed using Microarray Suite (MAS) version 5.0 (Affymetrix). When necessary, the data produced by MAS were exported into Microsoft Excel and further analyzed.

\section{Analysis of GeneChip data}

The MAS 5.0 signal was used as the basis for all subsequent analyses. Four independent biological replicates were conducted with the experimental and the reference samples. To identify genes differentially expressed with statistical significance, we used a combined criterion of expression ratios calculated by the averages of signal intensity and Student's $t$ test ( $P$ value). Gene transcripts with a ratio of induction of $>2$ or $<1 / 2$ in average signal intensity were regarded as upregulated or downregulated, respectively. Additionally, the expression calls of the MAS 5.0 software (present, P; marginal, M; or absent, A) were computed to give expression classes as follows: upregulated genes were those with an expression call of $\mathrm{P}$ in all the experimental samples; downregulated genes were those with an expression call of $\mathrm{P}$ for all the reference samples.

\section{Cytokinin measurements}

Cytokinins were extracted and purified as described previously (Dobrev and Kaminek 2002), with minor modifications. Cytokinins were extracted with methanol/ water/formic acid (75/20/5, by volume) containing labeled 
internal standards. The extracts were purified by OASIS HLB cartridges (Waters) and an OASIS MCX column (Waters). The cytokinin fractions were analyzed by low diffuse nanoflow liquid chromatography electrospray ionisation tandem mass spectrometry (LC-ESI-MS/MS). The packed nanocapillary columns integrated with nanosprayers were prepared in-house according to the previously reported procedure (Ishihama et al. 2002). An Inertsil ODS-3 C18 (3- $\mu \mathrm{m}$ particle size, $100-\AA$ pore size, GL Sciences) was used to supply packing materials. For details, see Supplemental Protocol 1.

\section{Results}

Changes in amino acid metabolism during the leaf development processes

In the vegetative stage, plants accumulate sugars and amino acids by photosynthesis and nitrogen assimilation in the leaves. These compounds are reallocated to developing organs and accumulated in the reproductive stages. To elucidate the regulatory mechanisms of amino acid metabolism during the leaf development process, we defined 4-, 5-, and 6-week-old Arabidopsis plants as stages 1, 2, and 3, respectively (Fig. 1a), and determined the amino acid contents of their leaves. Freshly emerged and expanded rosette leaves at each stage were selected for the analysis: the 8th and 9th true leaves were sampled in stage 1 and the 14th and 15th true leaves in stages 2 and 3. Under our culture conditions, yellowing-a marker of senescence-was not observed in these leaves.
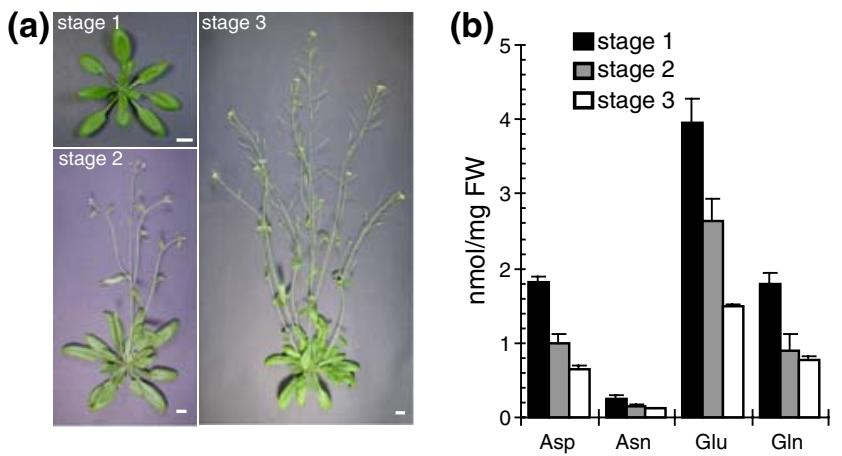

Fig. 1 Changes in leaf amino acid content during leaf-stage progression. a Stages of wild-type Arabidopsis thaliana (Col-0) plants. Plants grown on rockwool for 4 weeks (left), 5 weeks (center), and 6 weeks (right) were defined as stages 1,2, and 3, respectively. Bar $10 \mathrm{~mm}$. b Amino acid content in leaves at each stage. Plants of the wild-type (Col-0) were grown and freshly expanded rosette leaves (two leaves per plant) were harvested at midday and their amino acid contents determined. Values are mean $\pm \mathrm{SD}$ of average values of three plants
The contents of major amino acids in the leaf, such as glutamate, glutamine, and aspartate, decreased during leafstage progression (Fig. 1b). This result corresponded well to those of previous reports (Masclaux et al. 2000; Diaz et al. 2008). We used this alteration in amino acid profiling in a model experiment to elucidate the regulatory mechanisms of leaf amino acid metabolism.

Effects of removal of reproductive organs on metabolic regulation in leaves

The functions of source tissues in metabolism are regulated by signals released from the reproductive tissues (Nooden and Penney 2001). We investigated the effect of canceling these signals by removal of the reproductive tissues on metabolic regulation in leaves. In stage 2 , all organs above the rosette leaves were cut off and the amino acid contents of the leaves were determined $24 \mathrm{~h}$ after cutting treatment. This treatment was termed "excision of organs above the rosette leaves" (EOL); details are given in the "Materials and methods". For the detail analysis of sink-source regulation, excision of flower or silique is rational, whereas obvious effects were not detected. Then, it was assumed that EOL is better to examine a more dramatic effect inclusively.

The leaf contents of major amino acids had increased by $24 \mathrm{~h}$ after excision (Fig. 2a). The amino acid profile after the EOL treatment was almost the same as that before the bolting stage. There are two possibilities behind this change in the amino acid profile. One is that signals from the source organs affect the regulation of leaf amino acid metabolism. The other is that loss of the transportation (a)

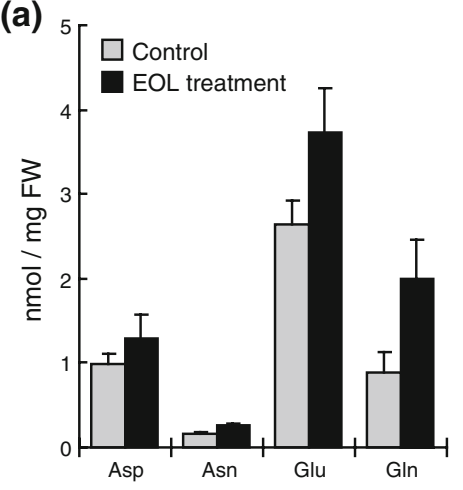

(b) ${ }^{20}[$ RBCS-1B/ACT2

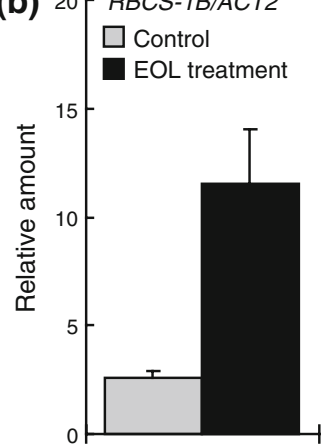

Fig. 2 Changes in leaf amino acid content and expression of RBCS$1 B$ after excision of organs above the rosette leaves (EOL). Stage 2 wild-type plants were treated by EOL, and freshly expanded rosette leaves (two leaves per plant) were harvested at midday $24 \mathrm{~h}$ after treatment. The content of major amino acids (a) and of RuBisCO small subunit 1B (RBCS-1B; At5g38430) mRNA (b) in the leaves were determined. Details of EOL and assay methods are described in the text. Values are mean \pm SD of three plants 
destination of amino acids as a nitrogen source simply caused the accumulation of amino acids in the leaves. Therefore, we investigated changes in gene expression to elucidate the possibility of the existence of regulatory mechanisms at the gene expression level. Expression of $R B C S-1 B$, which encodes a Rubisco small subunit and a marker of source activity, was drastically increased by $24 \mathrm{~h}$ after EOL treatment (Fig. 2b).

This result suggests that the presence of the reproductive organs affects the regulation of gene expression in the vegetative organs.

Correlation of changes in leaf gene expression between EOL treatment and stage progression

To investigate the mechanisms of the effects of EOL treatment and stage progression, we comprehensively analyzed gene expression using Affymetrix GeneChip technology. In the leaves, genes whose expression levels had changed during the transition from stage 1 to stage 3 and with EOL treatment were selected and plotted based on the ratio of their signal intensity (Fig. 3). The genes that showed increased (821 genes) and decreased (1,368 genes) expression during stage progression largely showed decreased and increased expression, respectively, after EOL treatment (Fig. 3a). In addition, the genes that showed increased (1,419 genes) and decreased (681 genes) expression after EOL treatment largely showed decreased and increased expression, respectively, during stage progression (Fig. 3b). These high negative correlations between stage progression and EOL treatment strongly suggested that the removal of sink organs containing the stems, flowers, siliques, fresh seeds, and young leaves led the gene expression in the rosette leaves to emulate globally that in the leaves of plants at younger stages. The expression of large numbers of DNA-replication- and celldivision-related genes was induced by EOL treatment and decreased during stage progression (data not shown), also suggesting that EOL treatment led to the emulation of a younger leaf-stage. Therefore, to investigate the mechanism of leaf metabolic regulation during leaf development, we took advantage of EOL treatment as part of a model experiment for monitoring metabolic regulation during leaf-stage progression and source-sink communication.

Induction of cytokinin-inducible genes by EOL treatment

Cytokinins are well-characterized plant hormones involved in leaf biological processes such as chlorophyll synthesis, nitrogen metabolism, and senescence (Ferreira and Kieber 2005; Sakakibara 2006). Therefore, we focused on changes in the expression of cytokinin-inducible genes. The
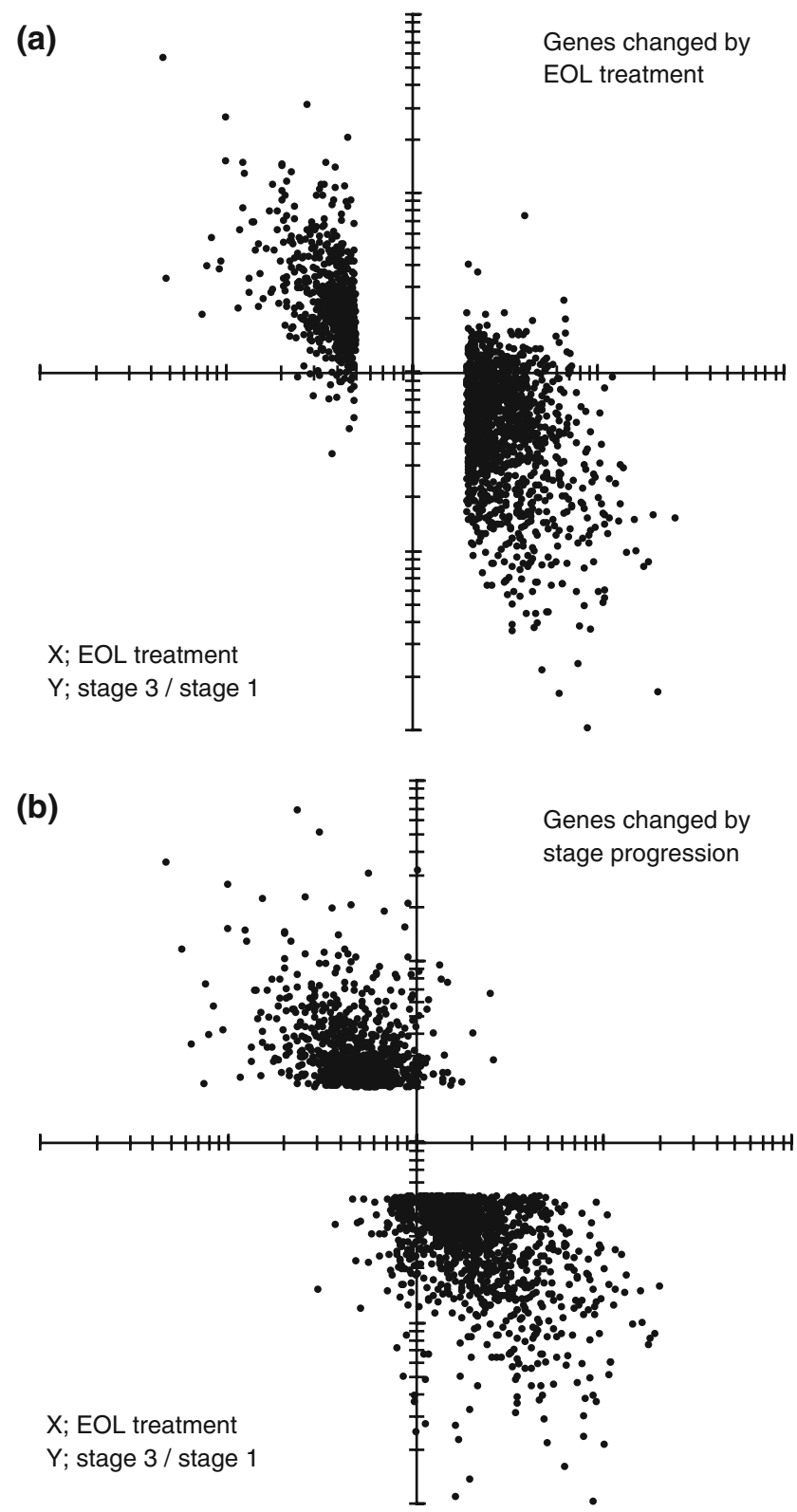

Fig. 3 Scatter plots of genes induced by excision of organs above the rosette leaves (EOL) and stage progression. Gene expression levels were determined by GeneChip analysis of each sample. Normalized fold expression $24 \mathrm{~h}$ after EOL treatment was plotted ( $x$-axis) against the normalized fold expression with stage progression ( $y$-axis; level at stage 3 divided by level at stage 1). Genes of which the expression level was changed by $>2$ or $<0.5(n=4, P<0.02)$ by EOL treatment (a), or by $>2$ or $<0.5(n=4, P<0.05)$ by stage progression (level at stage 3 divided by level at stage 1) (b) were selected and spotted. Method details are described in the text

expression of typical cytokinin-inducible genes (ARR4, $A R R 5, A R R 6, A R R 7$, and $C K X 4)$ was largely induced by EOL treatment and decreased during leaf-stage progression (Fig. 4). To confirm that these changes in gene expression were regulated by cytokinin signaling, we analyzed the responses in lines with knockout of the cytokinin receptors 

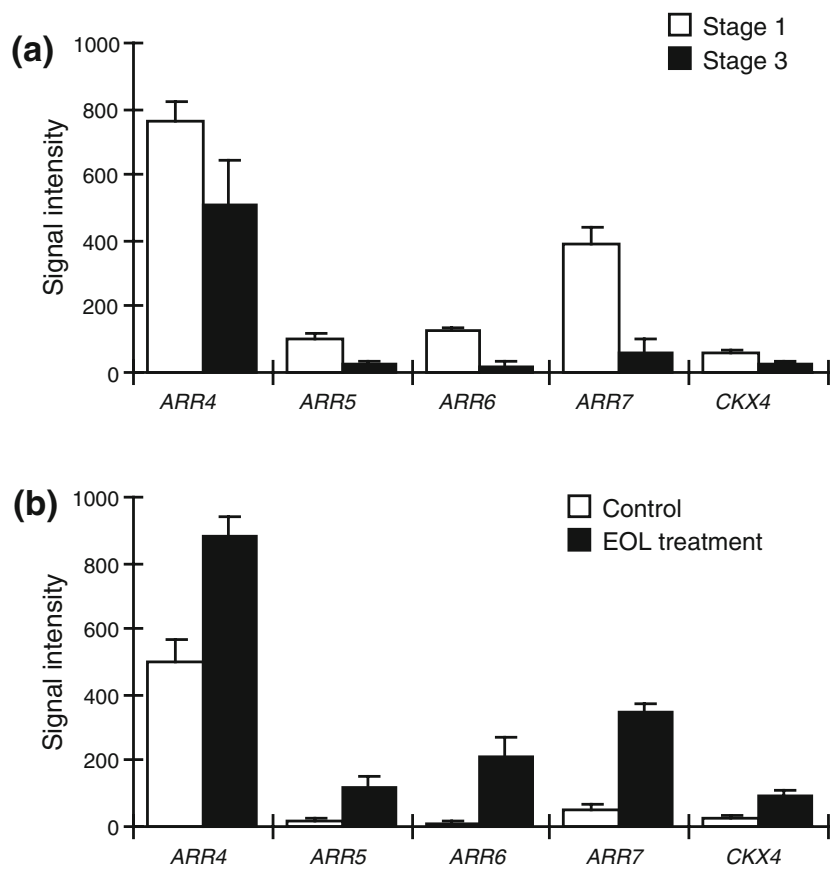

Fig. 4 Changes in expression levels of cytokinin-inducible genes. Expression levels of cytokinin-inducible genes were determined during stage progression (a) and after EOL treatment (b) by GeneChip analysis. Expression values are represented as signal intensity. The AGI codes of the genes were as follows: ARR4 (At1g10470), ARR5 (At3g48100), ARR6 (At5g62920), ARR7 (At1g19050), and $C K X 4$ (At4g29740). Presented values are mean \pm SD of average values of four plants

$A H K 2$ and $A H K 3$ (ahk2-2 and ahk3-3, respectively) (Higuchi et al. 2004). Because the timing of bolting differed between wild-type plants and the knockout lines (ahk2-2 was late and ahk3-3 was early under our conditions), the number of opened flowers was used as a marker of the developmental stage. Expression levels of the typical cytokinin-inducible genes $A R R 6$ and $A R R 7$ in the knockout lines after EOL treatment were lower than in the wild-type (Fig. 5). However, in both the wild-type and the knockout lines, the extent of the within-line changes in gene expression between the control and EOL treatments was similar, because the expression levels of these genes were lower in the untreated knockout lines than in the untreated wild-type. Two of three identified cytokinin receptors, AHK2 and $A H K 3$, are expressed in the above-ground tissues (Higuchi et al. 2004), and it is possible that functional redundancy of these genes decreased the differences between the knockout line and the wild-type after treatment in this experiment.

\section{Cytokinin biosynthesis IPT genes and cytokinin contents}

The induction of cytokinin-inducible genes by EOL treatment suggested that cytokinins were newly synthesized

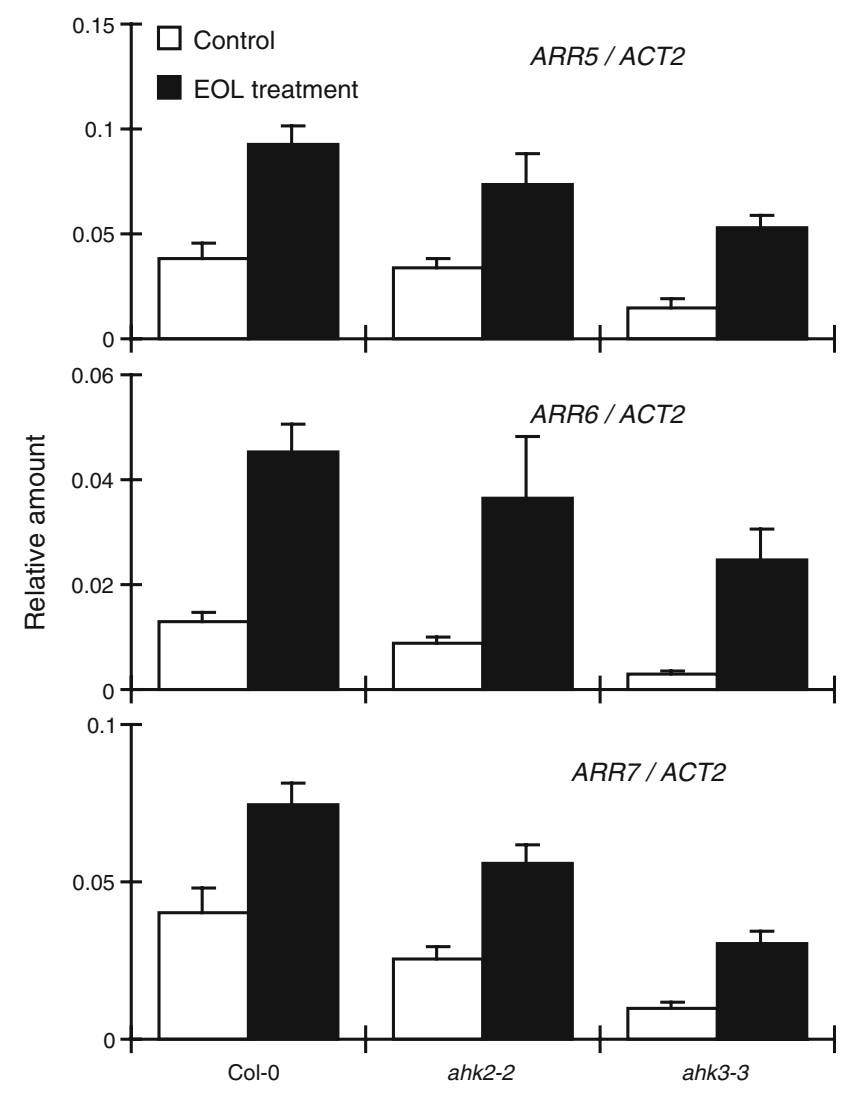

Fig. 5 Changes in expression levels of cytokinin-inducible genes in cytokinin receptor T-DNA insertion lines. Wild-type and T-DNA insertion lines (ahk2-2 and ahk3-3; Col-0 background) were grown for 5 weeks on rockwool and then treated by excision of organs above the rosette leaves (EOL). Freshly expanded rosette leaves (two leaves per plant) were harvested at midday after $24 \mathrm{~h}$ treatment. Expression levels of cytokinin-inducible genes (ARR5, ARR6, and ARR7) were determined by real-time PCR. Assay methods are described in the text. Presented values are mean $\pm \mathrm{SD}$ of average values of three plants

after treatment. To confirm the validity of this hypothesis, we determined the expression levels of the genes encoding cytokinin biosynthetic enzymes. The rate-limiting step of cytokinin biosynthesis in Arabidopsis is catalyzed by the ATP/ADP IPTs, Arabidopsis thaliana AtIPT1 and AtIPT4, and by their homologs AtIPT3, AtIPT5, AtIPT6, AtIPT7, and AtIPT8 (Kakimoto 2001; Miyawaki et al. 2004; Takei et al. 2001a). AtIPT2 and AtIPT9 are thought to function in tRNA maturation or modification (Takei et al. 2001a; Miyawaki et al. 2004). As we clearly observed changes in gene expression after EOL treatment in the main vein in our preliminary trials (data not shown), we harvested main veins $2,24 \mathrm{~h}$ after EOL treatment and determined the expression levels of the IPT genes by GeneChip. The level of expression of AtIPT3, which was the highest among the IPT genes in untreated plants, had increased to about $300 \%$ of the original, $2 \mathrm{~h}$ after EOL treatment (Table 1). Although the level of AtIPT9, which is thought to function 
Table 1 Expression of AtIPT genes

\begin{tabular}{|c|c|c|c|c|c|c|c|c|c|c|c|c|c|c|}
\hline \multirow[t]{4}{*}{ Gene } & \multirow[t]{4}{*}{ AGI code } & \multirow[t]{4}{*}{ GC id } & \multicolumn{6}{|l|}{$2 \mathrm{~h}$} & \multicolumn{6}{|l|}{$24 \mathrm{~h}$} \\
\hline & & & \multicolumn{4}{|c|}{ Raw signal } & \multicolumn{2}{|c|}{ Fold change } & \multicolumn{4}{|c|}{ Raw signal } & \multicolumn{2}{|c|}{ Fold change } \\
\hline & & & \multicolumn{2}{|l|}{ Exp. 1} & \multicolumn{2}{|l|}{ Exp. 2} & \multirow[t]{2}{*}{ Exp. 1} & \multirow[t]{2}{*}{ Exp. 2} & \multicolumn{2}{|l|}{ Exp. 1} & \multicolumn{2}{|l|}{ Exp. 2} & \multirow[t]{2}{*}{ Exp. 1} & \multirow[t]{2}{*}{ Exp. 2} \\
\hline & & & Control & EOL & Control & EOL & & & Control & EOL & Control & EOL & & \\
\hline AtIPT1 & At1g68460 & 260240_at & 3.8 & 5.6 & 3.8 & 2.3 & 1.5 & 0.6 & 3.8 & 8.7 & 0.9 & 0.8 & 2.3 & 0.9 \\
\hline AtIPT2 & At2g27760 & 266208_at & 23.7 & 38.6 & 14.0 & 28.5 & 1.6 & 2.0 & 23.3 & 21.7 & 19.7 & 24.2 & 0.9 & 1.2 \\
\hline AtIPT3 & At3g63110 & 251154_at & 53.1 & 181.2 & 44.1 & 108.9 & 3.4 & 2.5 & 54.6 & 68.5 & 87.4 & 65.2 & 1.3 & 0.7 \\
\hline AtIPT4 & At4g24650 & 254129_at & 3.6 & 6.4 & 1.8 & 6.8 & 1.8 & 3.8 & 6.5 & 1.4 & 1.2 & 7.6 & 0.2 & 6.3 \\
\hline AtIPT5 & At5g19040 & 249972_at & 1.3 & 0.9 & 3.3 & 4.3 & 0.7 & 1.3 & 2.4 & 1.1 & 0.7 & 0.7 & 0.5 & 1.0 \\
\hline AtIPT6 & At1g25410 & 255741_at & 3.2 & 1.5 & 1.2 & 0.8 & 0.5 & 0.7 & 1.2 & 1.3 & 1.1 & 0.3 & 1.1 & 0.3 \\
\hline AtIPT7 & At3g23630 & 258103_at & 0.5 & 3.1 & 1.2 & 0.3 & 6.2 & 0.2 & 0.8 & 0.5 & 0.9 & 0.4 & 0.6 & 0.4 \\
\hline AtIPT8 & At3g19160 & 257039_at & 3.8 & 7.2 & 10.1 & 6.6 & 1.9 & 0.7 & 10.6 & 11.7 & 11.6 & 4.9 & 1.1 & 0.4 \\
\hline AtIPT9 & At5g20040 & 246152_at & 48.6 & 36.9 & 42.1 & 27.6 & 0.8 & 0.7 & 49.6 & 91.7 & 32.6 & 114.0 & 1.8 & 3.5 \\
\hline
\end{tabular}

Wild-type plants were grown for 5 weeks and then treated by excision of organs above the rosette leaves (EOL). Expression levels of AtIPT genes in the main vein after EOL treatment were determined by GeneChip analysis. Experiments were repeated twice independently

in tRNA maturation or modification, had also increased by $24 \mathrm{~h}$ after treatment, the levels of the other genes had not changed markedly.

Induction of expression of the AtIPT3 gene suggested that EOL treatment induced AtIPT3-dependent cytokinin biosynthesis. To investigate the hypothesis that an increase in AtIPT3 gene expression is essential for the induction of cytokinin-inducible genes after EOL treatment, we determined the expression of these genes in the AtIPT3knockout plant atipt3-2 (Miyawaki et al. 2006). Although the expression of major cytokinin-inducible genes was induced by EOL treatment in both wild-type and atipt3-2 plants, the expression levels of all three genes in atipt3-2 were markedly lower than in the wild-type (Fig. 6). These results suggested that AtIPT3 mediates the metabolisms in the source leaves regulated by reproductive structures, but that there are likely to be some other factors involved in this regulation.

To investigate whether induction of the expression of AtIPT3 genes increased cytokinin levels, we measured cytokinin levels in the leaves and main vein of wild-type plant, $5 \mathrm{~h}$ after EOL treatment. Levels of the cytokinins tZR, iP, and isopentenyladenosine (iPR) markedly increased in both tissues after EOL treatment (Fig. 7). Although our low diffuse nanoflow LC-ESI-MS/MS systems did not quantify $\mathrm{tZ}$, which is an active form of cytokinin, because of its low concentration, the induction of expression of the cytokinin-inducible genes and the AtIPT3 gene and an increase in the $\mathrm{tZR}$, iPR, and iP contents indicated that cytokinin biosynthesis was activated after removal of the reproductive structures. Furthermore, the results suggested that the metabolic changes that occurred during leaf-stage progression are controlled by sink organs and are mediated by cytokinin signals.
$G D H 3$ is involved in amino acid biosynthesis downstream of the cytokinin signal

Because we found that cytokinin content increased after EOL treatment, we tried to identify the factors that control leaf amino acid content and are regulated by cytokinin signaling. To select the candidate genes, we screened the genes encoding amino acid biosynthesis from GeneChip data under EOL treatment (Table 2). Although the expression levels of almost all genes were not affected by EOL treatment, expression of one of the glutamate dehydrogenase (GDH) genes, $G D H 2$ and $G D H 3$, was markedly increased $2 \mathrm{~h}$ and $24 \mathrm{~h}$ after treatment, respectively. Analysis of the expression levels of $G D H 3$ after EOL treatment in lines with knockout of AHK3 (ahk3-3) and AtIPT3 (atipt3-2) revealed slight increase of expression of $G D H 3$ by EOL treatment in these knockout plants (Fig. 8a). Cytokinin treatment of the petioles of detached leaves of the wild-type induced $G D H 3$ expression. However, this induction of expression of $G D H 3$ genes in ahk3-3 occurred at very low levels (Fig. 8b). In contrast, $G D H 2$ was induced by EOL treatment in these knockout plants normally and not induced by cytokinin treatment, indicating that $G D H 2$ is not direct target of cytokinin signal. All of these data indicated that induction of $G D H 3$ expression after EOL treatment is regulated by cytokinin signals, suggesting that $G D H 3$ is a candidate enzyme for the control of cytokinin-mediated leaf nitrogen metabolism during leaf-stage progression.

\section{Discussion}

In the vegetative stage, plants assimilate inorganic nitrogen, which is absorbed by the root, by means of the light- 


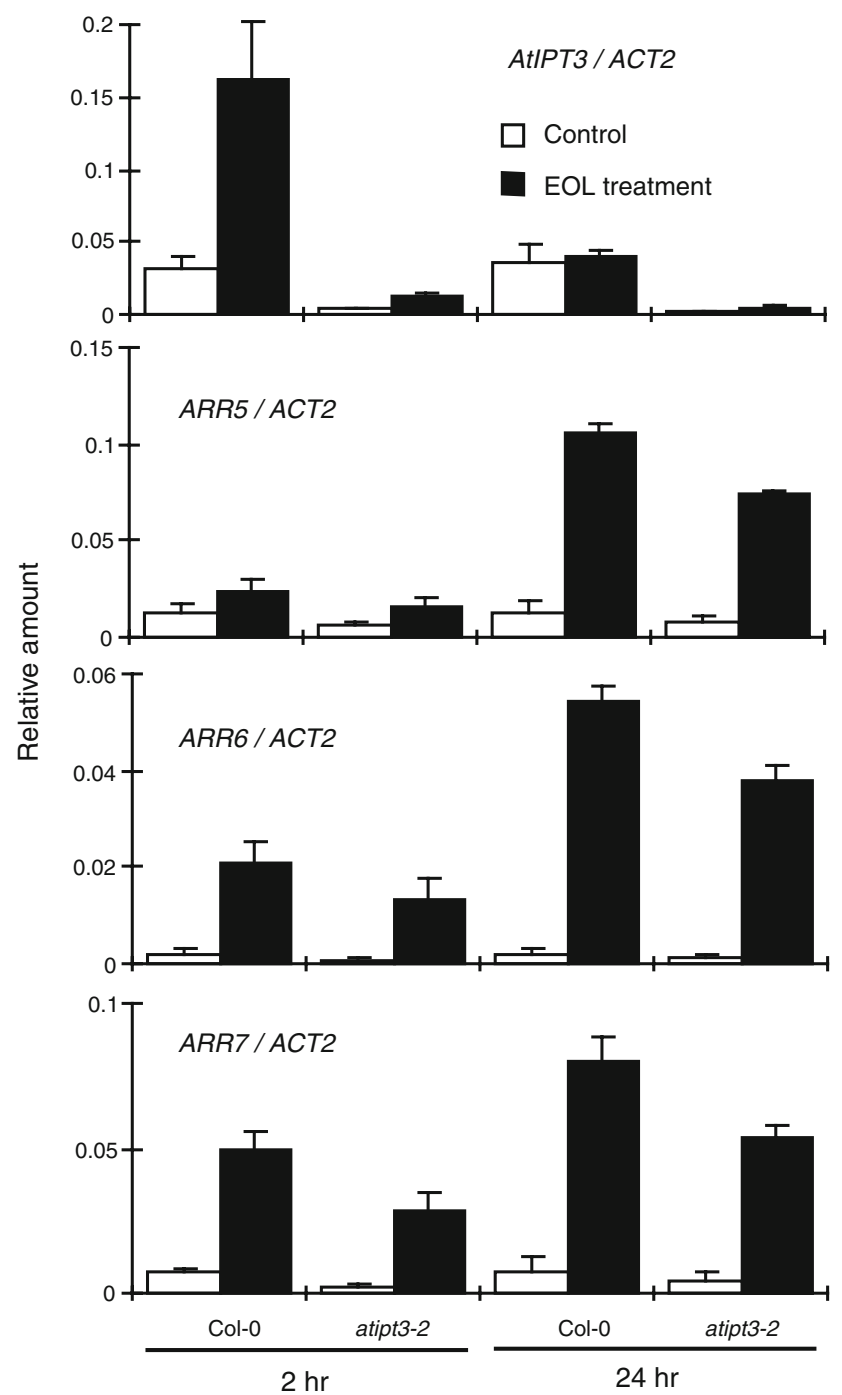

Fig. 6 Changes in expression levels of cytokinin-inducible genes in a line with insertion of AtIPT3 T-DNA (atipt3-2). Wild-type and atipt32 plants were grown for 5 weeks on rockwool and then treated with EOL. The main veins (in a ca. 5-mm-wide section containing the petiole) of freshly expanded rosette leaves (two leaves per plant) were harvested at midday after 2 or $24 \mathrm{~h}$. Expression levels of AtIPT3 and cytokinin-inducible genes (ARR5, ARR6, and ARR7) were determined by real-time PCR. Assay methods are described in the text. The presented values are mean \pm SD of average values of three plants

energy dependent GS/GOGAT cycle and GDH. Synthesized amino acids, such as glutamine and glutamate, are used to produce other amino acids, proteins, and nucleic acids. In the reproductive stage, these nitrogen compounds are depredated and the amino acids transported to sink tissues. Because the production of GS1 and AAPs, which are involved in whole plant partitioning of nitrogen, is induced in the reproductive stage (Miflin and Habash 2002; Tilsner et al. 2005), it is possible that expression of the genes encoding these enzymes is affected by the development of sink tissues. In this report, we observed a reduction

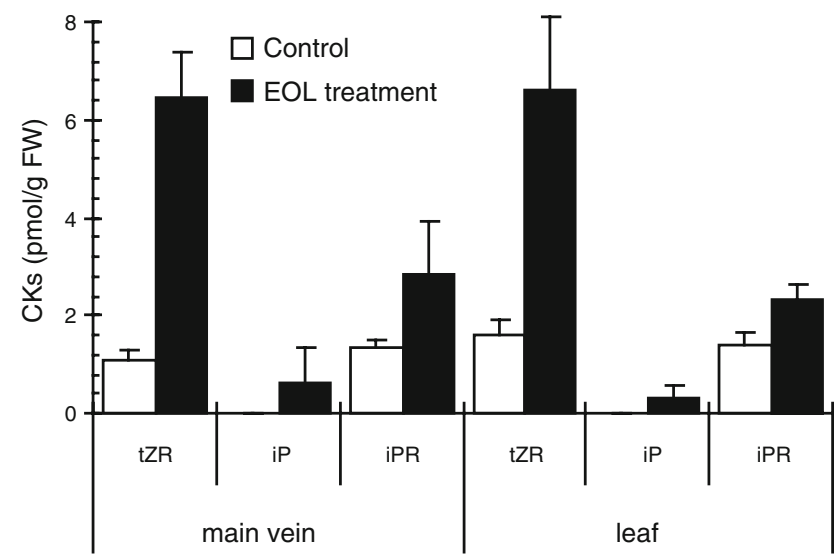

Fig. 7 Changes in cytokinin content. Wild-type plants were grown for 5 weeks on rockwool and then treated with EOL at midday. The main veins (in a ca. 5-mm-wide section containing the petiole) and leaf (minus the main vein) of freshly expanded rosette leave (two leaves per plant) were harvested $5 \mathrm{~h}$ after treatment. Cytokinin contents were quantified by low diffuse nanoflow LC-ESI-MS/MS. Assay methods are described in the text. The presented values are mean $\pm \mathrm{SD}$ of average values of four plants. $t Z R$ trans-zeatin riboside, $i P$ isopentenyladenine, $i P R$ isopentenyladenosine

in amino acid content during leaf-stage progression and an increase in amino acid content after removal of the reproductive structures, supporting these possibilities.

The decrease in the contents of major amino acids in the leaves during leaf-stage progression (Fig. 1b) indicated that nitrogen compounds were remobilized to the sink tissues during the reproductive developmental stage. This change in amino acid profiling has been reported previously (Masclaux et al. 2000; Diaz et al. 2008). Conversely, other reports have shown an increase in leaf asparagine content during senescence (Lin and $\mathrm{Wu}$ 2004; Lea et al. 2007). In these reports, asparagine was a major product of metabolism in senescing leaves, although these studies induced senescence by detachment of the leaf from the plant or by dark treatment, both of which are artificial processes that cancel sink-source communications.

The changes in amino acid profiles after removal of the reproductive structures could result from the loss of the destination for nutrient transport and wounding stress of the plant leaves. However, unlike removal of the reproductive structures, nitrogen supply by the roots did not induce dramatic changes in cytokinin-inducible gene expression (data not shown). Expression of wounding-inducible genes was not induced by EOL treatment (Supplementary Table 1). Wounding is generally mediated by jasmonate signals that increase in the senescing leaves (BuchananWollaston et al. 2003). Expression of almost all genes upregulated after EOL treatment was decreased during leaf-stage progression, indicating that the effects of EOL and jasmonate signaling mediation of the wounding response were independent. This information supported the 


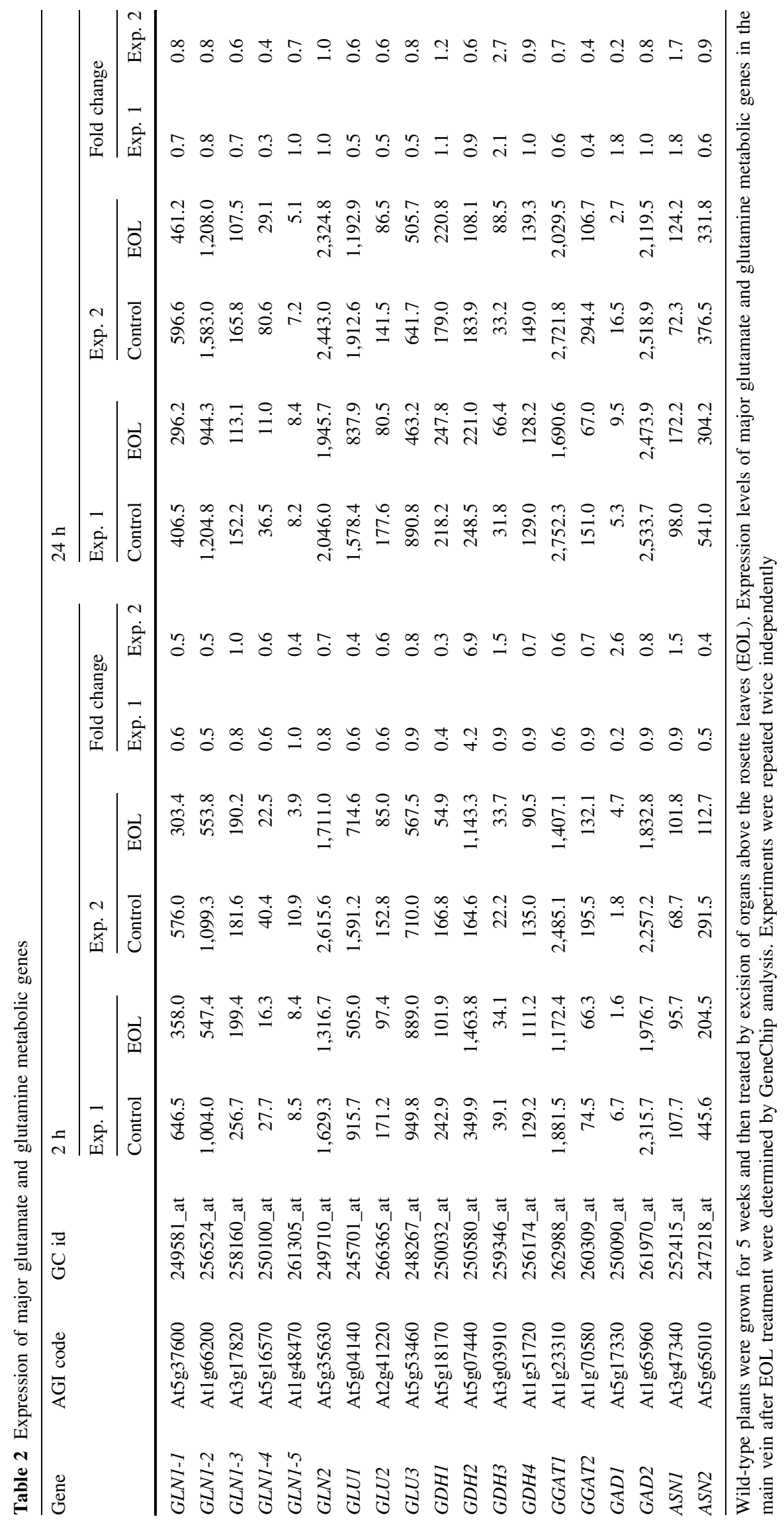



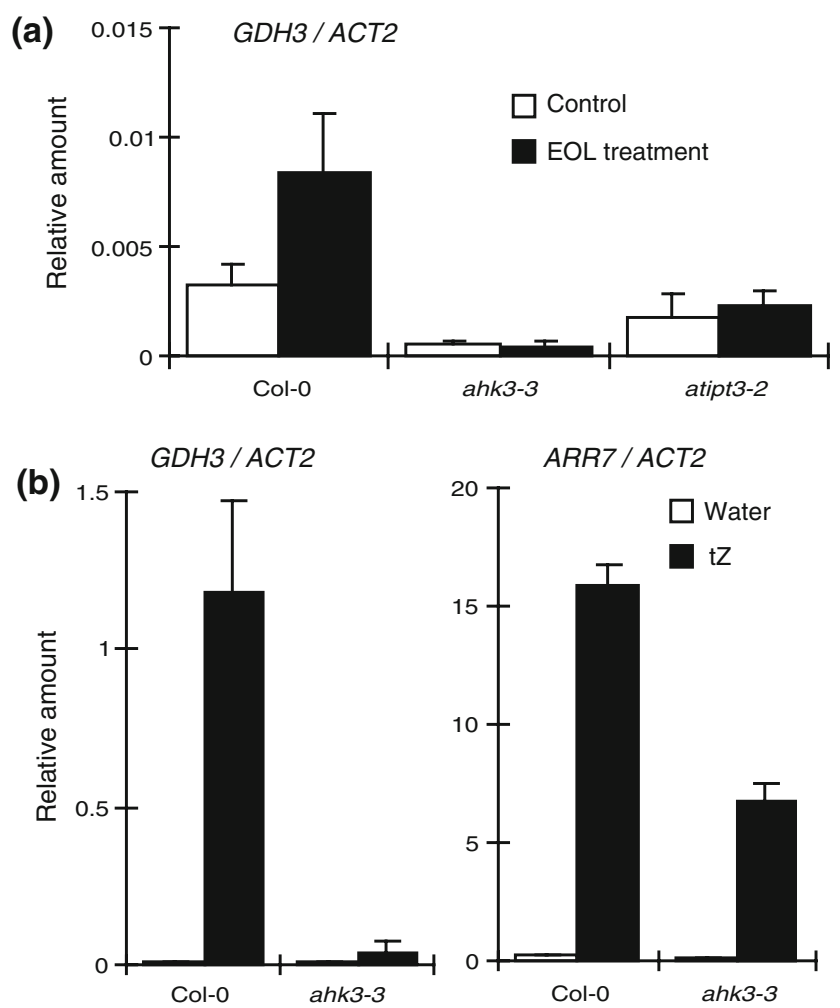

Fig. 8 Changes in expression levels of glutamate dehydrogenase (GDH3). a Wild-type and ahk3-3 and atipt3-2 plants were grown for 5 weeks on rockwool and then treated by EOL at midday. The main veins (in a ca. 5-mm-wide section containing the petiole) of freshly expanded rosette leaves (two leaves per plant) were harvested $24 \mathrm{~h}$ after treatment. b Wild-type and ahk3-3 plants were grown for 5 weeks on rockwool and the freshly expanded rosette leaves were then detached. At the site where the petiole was cut off, detached leaves were treated for $24 \mathrm{~h}$ with $20 \mu \mathrm{M}$ trans-zeatin (tZ); the main veins (in a ca. 5 -mm-wide section containing the petiole) were then harvested. Expression levels of GDH3 and $A R R 7$ were determined by real-time PCR. ARR7 is a control gene whose expression is induced by cytokinin. Assay methods are described in the text. Presented values are mean $\pm \mathrm{SD}$ of average values of three plants

hypothesis that the disappearance of some signals from the reproductive organs shifts the source leaves to younger stages in terms of the levels of gene expression and the amino acid profiles.

Removal of the reproductive structures induced global changes in gene expression. Expression of many genes normally expressed at higher levels in young leaves than in old leaves, as well as their amino acid profiles, changed to these of a younger stage after EOL treatment. In addition, the cytokinin content increased after treatment, suggesting that signals from the reproductive organs control the cytokinin-mediated metabolism of leaf-stage progression.

In pea (Pisum sativum L.), expression of PsIPT genes and cytokinin biosynthesis in the nodal stem are repressed by auxins and increased after decapitation (Tanaka et al. 2006). In Arabidopsis, it was possible that removal of the reproductive structures repressed auxin signals in the leaves. To explore this possibility, we examined the effect of auxin treatment by quantifying auxin-inducible gene expression. Expression of AtIPT3 was slightly repressed by auxin treatment (Supplementary Fig. 1), and auxin-inducible gene expression was not affected by removal of the reproductive organs (Supplementary Table 1). These results suggested that auxin signals from the apical tissues are not essential in the control of leaf metabolic profiles mediated by cytokinin biosynthesis.

Levels of $G D H 3$ increased after removal of the reproductive structures, whereas in lines with knockout of cytokinin biosynthesis (atipt3-2) and a cytokinin receptor (ahk3-3), upregulation of $G D H 3$ was not observed. These observations suggest that the cytokinin-GDH3 pathway could control amino acid metabolism during leaf-stage progression. Analysis of $G D H 3$-knockout plants led us to confirm that $G D H 3$ is essential for the regulation of leaf amino acid metabolism during development of the reproductive organs.

After removal of the reproductive structures, no differences in amino acid profiles between the wild-type and atipt3-2 were observed (data not shown). The amino acid contents of the source tissues are therefore controlled strictly not only by cytokinin-GDH3 pathways, but also by multiple regulation systems. After EOL treatment of atipt32 plants, GDH3 induction disappeared, whereas the expression of many other genes whose expression was induced by EOL and decreased by stage progression was induced (data not shown). These observations indicated that cytokinin signaling is partly involved in the sinksource regulation of gene expression and metabolism. Indeed, some plant hormones, such as ethylene and jasmonate, are known to regulate leaf senescence and its metabolism (Buchanan-Wollaston et al. 2003), and the amino acid profile is controlled by some metabolic pathway, suggesting that cancellation of the cytokinin signal alone is not sufficient for the complete control of leaf metabolism. We expect that further use of EOL in model experiments will help to identify additional key molecules involved in source-sink regulation.

Cytokinins, whose content is increased by nitrate treatment in the root, function as messengers of nitrogen metabolism (Takei et al. 2001b, 2004), whereas cytokinininducible genes that function as regulators of nitrogen metabolism have not been identified. Transgenic plants that express IPT genes under the control of senescence-inducible promoters show delayed chlorophyll degradation and increased productivity (Gan and Amasino 1995; McCabe et al. 2001). The fact that induction of expression of $G D H 3$ was dependent on cytokinins suggested that the cytokinin signal functions as a regulator of leaf nitrogen metabolism and productivity. GDH (EC 1.4.1.2) catalyzes a reversible 
reaction for the reductive amination of 2-oxoglutarate to form glutamate in the presence of the cofactor $\mathrm{NAD}(\mathrm{P}) \mathrm{H}$. Analyses of GDH-mutant plants and incorporation of labeled ammonium in Arabidopsis and tobacco have suggested that GDH catalyses deamination of glutamate under normal or carbon-deficient conditions and amination of 2oxoglutarate under high salt- or nitrogen-stress conditions (Melo-Oliveira et al. 1996; Masclaux-Daubresse et al. 2006; Skopelitis et al. 2006, 2007; Miyashita and Good 2008). In Arabidopsis, there are three NADH-dependent GDH genes (GDH1, At5g18170; GDH2, At5g07440; GDH3, At3g03910). Analysis of mutant (gdhl-1) phenotypes and the expression of $G D H 1$ indicate that $G D H 1$ plays a role in ammonium assimilation under high inorganic nitrogen conditions (Melo-Oliveira et al. 1996). Analysis of plants with knockout of the $G D H 1$ and $G D H 2$ genes suggests that GDH plays a central role in amino acid breakdown under carbon-deficient conditions (Miyashita and Good 2008). GDH activity was negligible in both the shoots and roots of 17-day-old double-knockout mutants, suggesting that $G D H 3$ plays only a minor role in these tissues of young plants and under normal growing conditions.

Tissue-specific expression analysis by using reporter genes indicates that $G D H 3$, whose expression is low in the leaf, is expressed in the leaf veins. The induction of $G D H 3$ by cytokinin treatment was restricted to within the leaf vein (Supplementary Fig. 2a, c). Expression of GDH3 was induced after EOL and cytokinin treatment, and the induction disappeared in atipt3-2 and ahk3-3 plants, suggesting that cytokinins play a role in the regulation of amino acid metabolism by GDH3; as well as in the vein, specific expression of $G D H 3$ was observed in the lateral root primordia (Supplementary Fig. 2b), suggesting that $G D H 3$ expression is controlled by cytokinins. The contribution of $\mathrm{GDH} 3$ to cytokinin-mediated nitrogen metabolism is unclear. Therefore, it will be important to analyze the effect of cytokinin application to the leaves on the phenotypes of $G D H 3$-knockout plants.

In this study, we succeeded in determining that cytokinin synthesis is an important event in the changes induced in the leaf by development of the reproductive organs. However, it is unclear what regulates leaf cytokinin synthesis. Elucidation of the regulatory mechanisms of source activities controlled by sink organs would help to produce useful crops with high productivity.

\footnotetext{
Acknowledgments Part of this work was performed in the project "Development of Fundamental Technologies for Controlling the Production of Industrial Materials by Plants," which was supported by the New Energy and Industrial Technology Development Organization (NEDO), Japan. The authors are grateful to Tatsuo Kakimoto (Osaka University, Japan) for providing the knockout plants and helpful discussions and to Tsuyoshi Nakagawa (Shimane University,
}

Japan) for providing the Gateway plasmid for reporter gene assay. We also thank Kiyoshi Miwa, Akira Mitsui, Hiroyuki Kiyohara, and Ryoko Nagasaka for their helpful discussions and technical assistance.

Open Access This article is distributed under the terms of the Creative Commons Attribution Noncommercial License which permits any noncommercial use, distribution, and reproduction in any medium, provided the original author(s) and source are credited.

\section{References}

Bowsher CG, Lacey AE, Hanke GT, Clarkson DT, Saker LR, Stulen I, Emes MJ (2007) The effect of Glc6P uptake and its subsequent oxidation within pea root plastids on nitrite reduction and glutamate synthesis. J Exp Bot 58:1109-1118

Brenner WG, Romanov GA, Köllmer I, Bürkle L, Schmülling T (2005) Immediate-early and delayed cytokinin response genes of Arabidopsis thaliana identified by genome-wide expression profiling reveal novel cytokinin-sensitive processes and suggest cytokinin action through transcriptional cascades. Plant $\mathbf{J}$ 44:314-333

Buchanan-Wollaston V, Earl S, Harrison E, Mathas E, Navabpour S, Page T, Pink D (2003) The molecular analysis of leaf senescence-a genomics approach. Plant Biotechnol J 1:3-22

Diaz C, Lemaître T, Christ A, Azzopardi M, Kato Y, Sato F, MorotGaudry JF, Le Dily F, Masclaux-Daubresse C (2008) Nitrogen recycling and remobilization are differentially controlled by leaf senescence and development stage in Arabidopsis under low nitrogen nutrition. Plant Physiol 147:1437-1449

Dobrev PI, Kaminek M (2002) Fast and efficient separation of cytokinins from auxin and abscisic acid and their purification using mixed-mode solid-phase extraction. J Chromatogr A 950:21-29

Ferreira FJ, Kieber JJ (2005) Cytokinin signaling. Curr Opin Plant Biol 8:518-525

Gan S, Amasino RM (1995) Inhibition of leaf senescence by autoregulated production of cytokinin. Science 270:1986-1988

Haberer G, Kieber JJ (2002) Cytokinins. New insights into a classic phytohormone. Plant Physiol 128:354-362

Higuchi M, Pischke MS, Mahonen AP, Miyawaki K, Hashimoto Y, Seki M, Kobayashi M, Shinozaki K, Kato T, Tabata S, Helariutta Y, Sussman MR, Kakimoto T (2004) In planta functions of the Arabidopsis cytokinin receptor family. Proc Natl Acad Sci USA 101:8821-8826

Hirose N, Takei K, Kuroha T, Kamada-Nobusada T, Hayashi H, Sakakibara H (2008) Regulation of cytokinin biosynthesis, compartmentalization and translocation. J Exp Bot 59:75-83

Ireland RJ, Lea PJ (1999) The enzymes of glutamine, glutamate, asparagine, and aspartate metabolism. In: Singh BK (ed) Plant amino acids: biochemistry and biotechnology. Marcel Dekker, New York, pp 49-109

Ishihama Y, Rappsilber J, Andersen JS, Mann M (2002) Microcolumns with self-assembled particle frits for proteomics. J Chromatogr A 979:233-239

Kakimoto T (2001) Identification of plant cytokinin biosynthetic enzymes as dimethylallyl diphosphate:ATP/ADP isopentenyltransferases. Plant Cell Physiol 42:677-685

Kakimoto T (2003) Perception and signal transduction of cytokinins. Annu Rev Plant Biol 54:605-627

Kim HJ, Ryu H, Hong SH, Woo HR, Lim PO, Lee IC, Sheen J, Nam HG, Hwang I (2006) Cytokinin-mediated control of leaf 
longevity by $A H K 3$ through phosphorylation of $A R R 2$ in Arabidopsis. Proc Natl Acad Sci USA 103:814-819

Lea PJ, Miflin BJ (2003) Glutamate synthase and the synthesis of glutamate in plants. Plant Physiol Biochem 41:555-564

Lea PJ, Sodek L, Parry MAJ, Shewry PR, Halford NG (2007) Asparagine in plants. Ann Appl Biol 150:1-26

Leegood RC, Lea PJ, Adcock MD, Hausler RE (1995) The regulation and control of photorespiration. J Exp Bot 46:1397-1414

Lin JF, Wu SH (2004) Molecular events in senescing Arabidopsis leaves. Plant J 39:612-628

Masclaux C, Valadier MH, Brugiere N, Morot-Gaudry JF, Hirel B (2000) Characterization of the sink/source transition in tobacco (Nicotiana tabacum L.) shoots in relation to nitrogen management and leaf senescence. Planta 211:510-518

Masclaux-Daubresse C, Reisdorf-Cren M, Pageau K, Lelandais M, Grandjean O, Kronenberger J, Valadier MH, Feraud M, Jouglet T, Suzuki A (2006) Glutamine synthetase-glutamate synthase pathway and glutamate dehydrogenase play distinct roles in the sink-source nitrogen cycle in tobacco. Plant Physiol 140:444-456

McCabe MS, Garratt LC, Schepers F, Jordi WJ, Stoopen GM, Davelaar E, van Rhijn JH, Power JB, Davey MR (2001) Effects of P(SAG12)-IPT gene expression on development and senescence in transgenic lettuce. Plant Physiol 127:505-516

Melo-Oliveira R, Oliveira IC, Coruzzi GM (1996) Arabidopsis mutant analysis and gene regulation define a nonredundant role for glutamate dehydrogenase in nitrogen assimilation. Proc Natl Acad Sci USA 93:4718-4723

Miflin BJ, Habash DZ (2002) The role of glutamine synthetase and glutamate dehydrogenase in nitrogen assimilation and possibilities for improvement in the nitrogen utilization of crops. J Exp Bot 53:979-987

Miyashita Y, Good AG (2008) NAD(H)-dependent glutamate dehydrogenase is essential for the survival of Arabidopsis thaliana during dark-induced carbon starvation. J Exp Bot 59:667-680

Miyawaki K, Matsumoto-Kitano M, Kakimoto T (2004) Expression of cytokinin biosynthetic isopentenyltransferase genes in Arabidopsis: tissue specificity and regulation by auxin, cytokinin, and nitrate. Plant J 37:128-138

Miyawaki K, Tarkowski P, Matsumoto-Kitano M, Kato T, Sato S, Tarkowska D, Tabata S, Sandberg G, Kakimoto T (2006) Roles of Arabidopsis ATP/ADP isopentenyltransferases and tRNA isopentenyltransferases in cytokinin biosynthesis. Proc Natl Acad Sci USA 103:16598-16603

Noguchi Y, Zhang QW, Sugimoto T, Furuhata Y, Sakai R, Mori M, Takahashi M, Kimura T (2006) Network analysis of plasma and tissue amino acids and the generation of an amino index for potential diagnostic use. Am J Clin Nutr 83:513S-519S

Nooden LD (1980) Senescence in the whole plant. In: Thimann KV (ed) Senescence in plants. CRC Press, Boca Raton, pp 219-258

Nooden LD, Penney JP (2001) Correlative controls of senescence and plant death in Arabidopsis thaliana (Brassicaceae). J Exp Bot 52:2151-2159
Sakakibara H (2006) Cytokinins: activity, biosynthesis, and translocation. Annu Rev Plant Biol 57:431-449

Sakakibara H, Takei K, Hirose N (2006) Interactions between nitrogen and cytokinin in the regulation of metabolism and development. Trends Plant Sci 11:440-448

Skopelitis DS, Paranychianakis NV, Kouvarakis A, Spyros A, Stephanou EG, Roubelakis-Angelakis KA (2007) The isoenzyme 7 of tobacco $\mathrm{NAD}(\mathrm{H})$-dependent glutamate dehydrogenase exhibits high deaminating and low aminating activities in vivo. Plant Physiol 145:1726-1734

Skopelitis DS, Paranychianakis NV, Paschalidis KA, Pliakonis ED, Yakoumakis D, Delis ID, Kouvarakis A, Papadakis AK, Stephanou E, Roubelakis-Angelakis KA (2006) Abiotic stress generated ROS signal expression of anionic glutamate dehydrogenases to form glutamate for proline synthesis. Plant Cell $18: 2767-2781$

Takei K, Sakakibara H, Sugiyama T (2001a) Identification of genes encoding adenylate isopentenyltransferase, a cytokinin biosynthesis enzyme, in Arabidopsis thaliana. J Biol Chem 276:2640526410

Takei K, Sakakibara H, Taniguchi M, Sugiyama T (2001b) Nitrogendependent accumulation of cytokinins in root and the translocation to leaf: implication of cytokinin species that induces gene expression of maize response regulator. Plant Cell Physiol 42:85-93

Takei K, Ueda N, Aoki K, Kuromori T, Hirayama T, Shinozaki K, Yamaya T, Sakakibara H (2004) AtIPT3 is a key determinant of nitrate-dependent cytokinin biosynthesis in Arabidopsis. Plant Cell Physiol 45:1053-1062

Tanaka M, Takei K, Kojima M, Sakakibara H, Mori H (2006) Auxin controls local cytokinin biosynthesis in the nodal stem in apical dominance. Plant J 45:1028-1036

Tatematsu K, Ward S, Leyser O, Kamiya Y, Nambara E (2005) Identification of cis-elements that regulate gene expression during initiation of axillary bud outgrowth in Arabidopsis. Plant Physiol 138:757-766

Tilsner J, Kassner N, Struck C, Lohaus G (2005) Amino acid contents and transport in oilseed rape (Brassica napus L.) under different nitrogen conditions. Planta 221:328-338

To JP, Kieber JJ (2007) Cytokinin signaling: two-components and more. Trends Plant Sci 13:85-92

Yamaya T, Oaks A (2004) Metabolic regulation of ammonium uptake and assimilation. In: Amâcio S, Stulen I (eds) Nitrogen acquisition and assimilation in higher plants. Kluwer, Dordrecht, pp 35-63

Yamaya T, Obara M, Nakajima H, Sasaki S, Hayakawa T, Sato T (2002) Genetic manipulation and quantitative-trait loci mapping for nitrogen recycling in rice. J Exp Bot 53:917-925 